\title{
Active stabilization in robotic vision systems
}

\author{
Vladimir Serebrennyi, Andrei Boshliakov and Georgii Ovsiankin * \\ Bauman Moscow State Technical University, Mechanical Engineering Department, 105005 ul. Baumanskaya 2-ya, 5, Moscow, Russia
}

\begin{abstract}
In the article considered prospective approaches to the design of active systems for stabilizing systems based on the parallel kinematics mechanism and possible applications of such systems. Attention is drawn to the fact that not only object fluctuations are an important object for stabilization, but it is also important to compensate for the body vibrations, along with its vibrations. Based on the analysis, it was concluded that it is perspective to use mechanisms with parallel kinematics for the design of active stabilization systems. Was obtained a mathematical model of the hexapod, according to which a computer model in the Simulink package was designed. Its analysis confirmed the possibility of using a mechanism with parallel kinematics in designing an active stabilization system and presented requirements to the actuators of the system.
\end{abstract}

\section{Introduction}

At present, optical systems are widely used to analyse and assess the current situation on the ground. At the same time, the requirements imposed on the quality of the image obtained from the equipment on such systems have increased. The requirements for the accuracy of optical systems can reach units of angular minutes. As a consequence, the task of stabilization comes first. The field of application of systems, where high demands are placed on the quality of the information received, is quietly wide.

\section{Existing approaches}

In remote earth sensing systems, high demands are placed on the quality of the resulting image. This is due to the growing popularity of automated intelligent analysis of aerospace information. The moving parts of the spacecraft create structural vibrations, which, acting on the mirror, degrade the quality of the resulting image [1].

In the aero cartography problem perspective approaches are (Fig. 1):

- Laser scanning

- Creation of digital models of terrain and objects

- Photographing

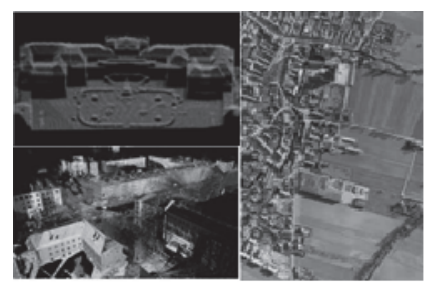

Fig. 1. Laser scanning. Creation of digital models of terrain and objects.
A serious impact on the quality of the resulting image is due to the housing vibrations generated by the engine operation. Vibration data due to the high altitude of the flight of the aircraft $(100 \ldots 2500 \mathrm{~m})$ lead to a shift in the pixels of the CCD matrix of photo equipment installed on board the aircraft. According to GOST 26382-84, the permissible levels of vibro-displacement lie in the interval $63 \ldots 437 \mathrm{um}$ at frequencies of $20 \ldots 100 \mathrm{~Hz}$. Therefore, it is possible to form the following requirements for advanced high-precision stabilization systems

- accuracy of stabilization - units of angular seconds

- range of operating frequencies - up to a hundred hertz

- stabilization in three axes

Actual question is increasing the accuracy of existing systems, both by designing new ones, and increasing the accuracy of the existing ones. An important part of the stabilization problem is not only compensation of the body oscillations, but also of its vibrations. For this task, a perspective approach is to use the mechanisms of parallel kinematics, which make it possible to design an active system of three-dimensional stabilization. At the moment, studies are underway to build stabilization systems that differ in the principle of operation, the number of stabilized axes, the limiting values of deviations and other characteristics.

Design of a stabilization system for radio telescope with segment mirrors [1] (Fig. 2).

The diameter of each mirror is about 3 meters, on each of them is installed a system of passive vibration damping. Tuned mass dampers (TMDs) were selected to mitigate the effects of the Segmented Mirror Telescope segment vibration modes because of their ability to add significant damping to resonant frequencies as add-on passive devices without changing the original optical load path. These devices have previously been shown to be effective on telescope structures with image jitter due to resonant

Corresponding author: george.ovciankin@gmail.com 
vibration. Figure 3 shows the effect in the frequency domain of a typical TMD on a resonant vibration mode of an idealized structure, along with the associated time domain response with and without the TMD.

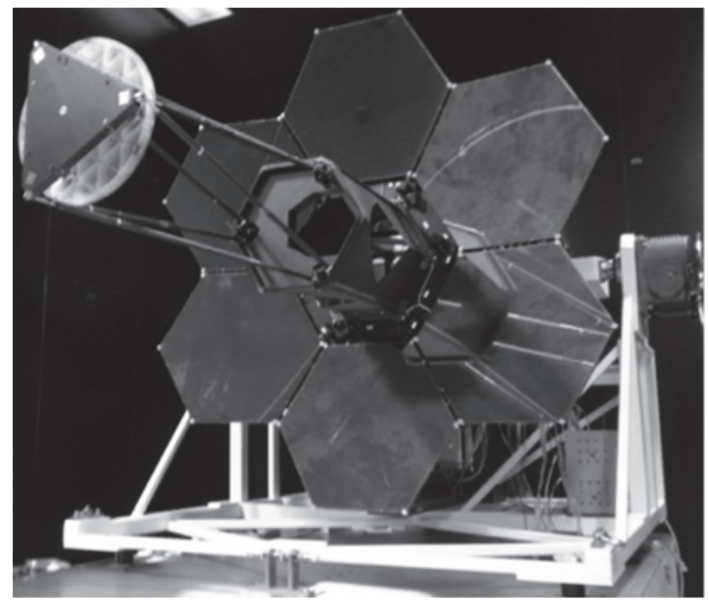

Fig. 2. Segmented Mirror Telescope.
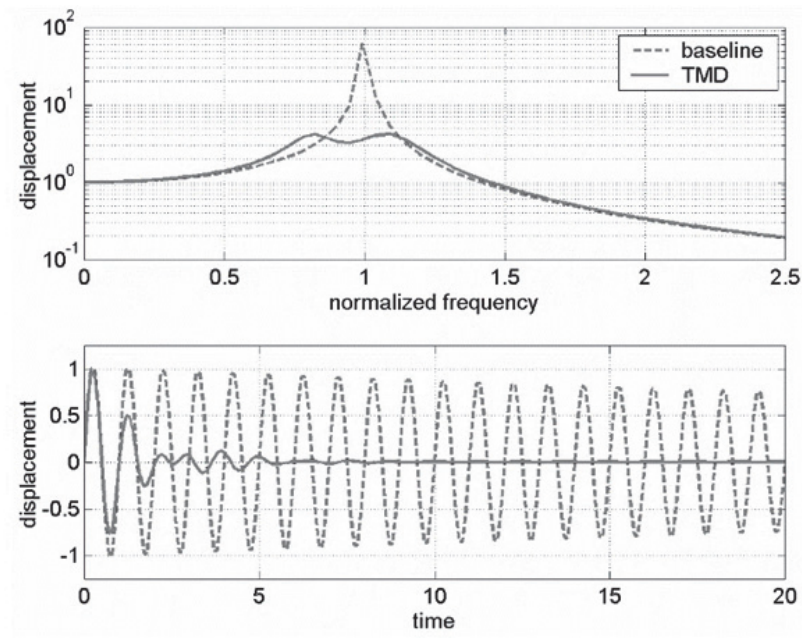

Fig. 3. Comparison of frequency and time domain responses with and without TMD.

Within work [2] a model of a 6 DOF (degree-offreedom) vibration isolation system with semi-active control, using magnetorheological (MR) technology is investigated. The geometry of the parallel platform is determined in a way which maximizes the Quality Index. Parallel platform mechanisms are ideal candidates for 6 DOF positioning and vibration isolation. A 6 DOF parallel platform which utilizes semi-active vibration control has not received as much attention as its passive and active counterparts have. Each leg of the platform is modelled as a two DOF system with a magnetorheological (MR) damper for adjustable damping. The class of semi-active drives is considered in detail in [3]. a)

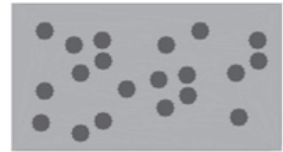

b)

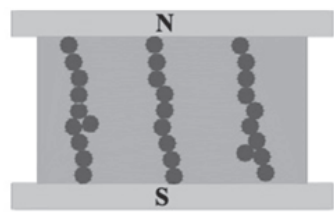

Fig. 4. Magnetorheological fluid: a) no magnetic field, b) with magnetic field.
The authors analysed the design of a parallel manipulator and selected a model for the legs (Fig. 5) and analysed the stabilizing properties of the received leg and semi-active control (Fig. 6).

a)

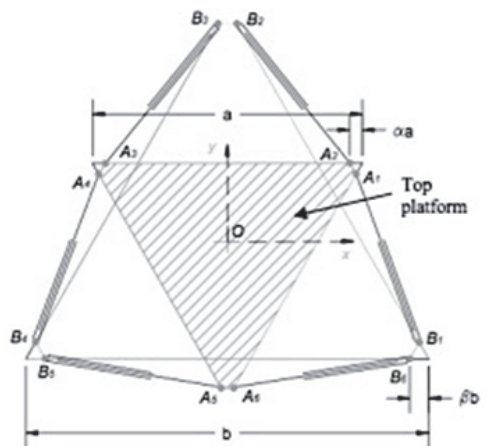

b)

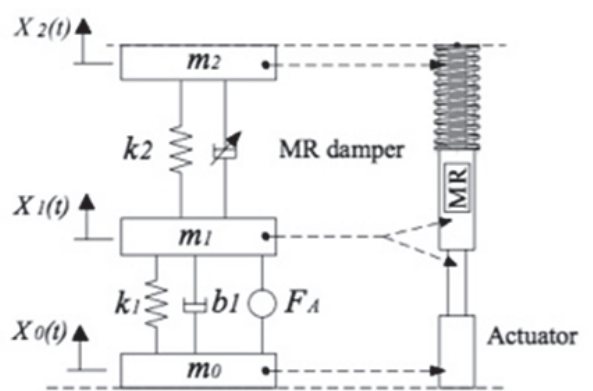

Fig. 5. Two DOF platform leg model with MR damper.

The MR damper was modelled in Simulink. The input to the model is the relative velocity between the two masses which the damper connects and the control current. In the simulations, the MR damper is assumed to respond very fast and the time delay associated with the damper's response time is ignored.

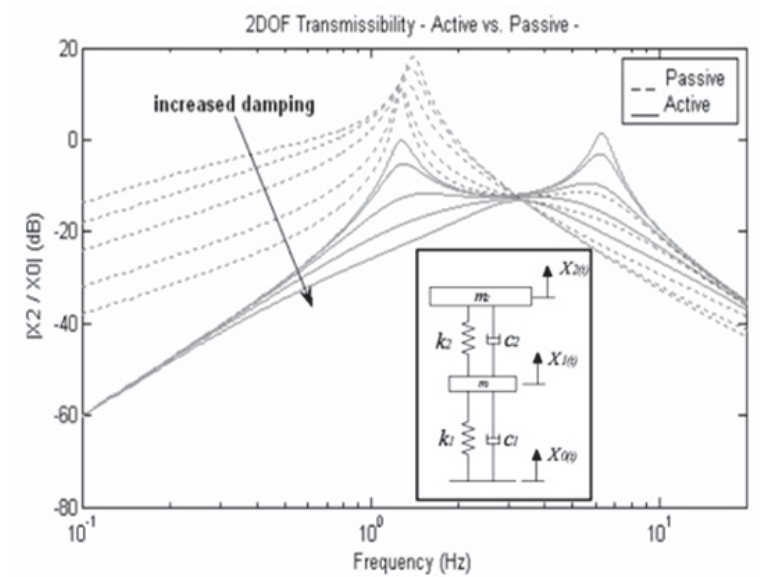

Fig. 6. Transmissibility of the two DOF mass-spring- damper system with passive and active skyhook damper control

Papers $[4,5]$ disclose a stiff active interface wherein a six degree-of-freedom Stewart platform, a standard hexapod with a cubic architecture, is used to actively increase the structural damping of flexible systems attached to it. It can also be used to rigidly connect arbitrary substructures while damping them.

Each leg of the active interface consists of a linear 
piezo electric actuator, a collocated force sensor and flexible tips for the connections with the two end plates. By providing the legs with strain or elongation sensors, this active interface can also be used as an interface with infinite stiffness at low frequency (i.e. for machine tools), a 6 DOF positioning and steering device for space applications as well as a micro vibration isolator (Fig. 7).

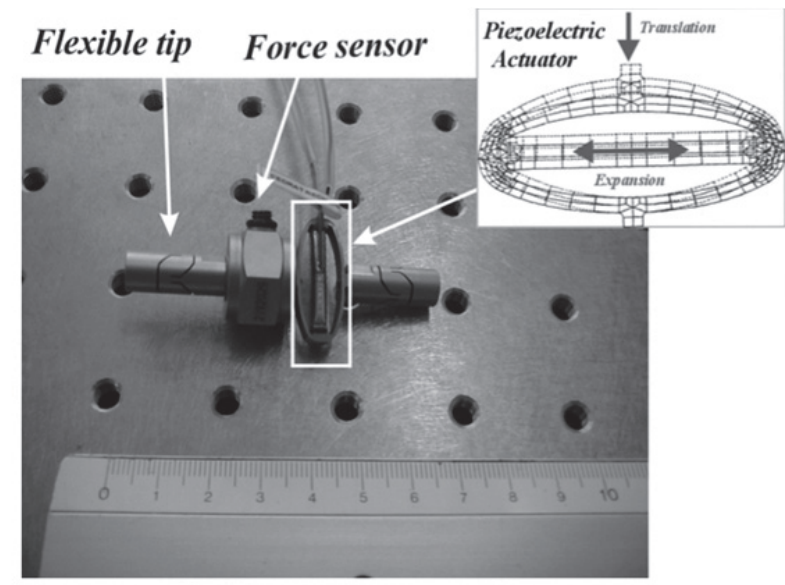

Fig. 7. The active leg assembly of Stewart platform.

To avoid the problems of friction and backlash in the joints, flexible tips were used instead of spherical joints.

These flexible tips have zero friction, zero backlash, high axial stiffness and relatively low bending stiffness. It will be shown that the existence of this bending stiffness makes a limitation for the control authority because it shifts the transmission zeroes to a higher frequency, which will decrease the damping effect expected from each closed-loop pole. (Fig. $8 \mathrm{a}, \mathrm{b}$ ).
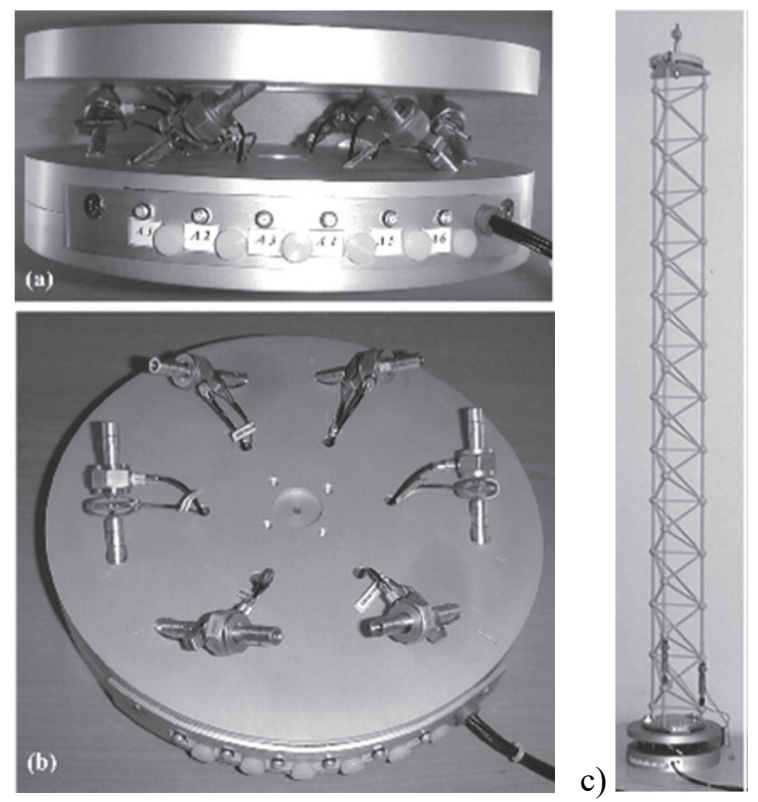

Fig. 8. The Stewart platform (a): complete hexapod, (b): the hexapod with the upper plate removed, (c): experimental setup.

In order to evaluate the damping performances of the interface, the hexapod is connected to a flexible payload, a $150 \mathrm{~cm}$ long steel truss structure (Fig. $8 \mathrm{c}$ ). A decentralized integral force feedback control scheme was applied to the experimental system. The six independent controllers have been implemented on a DSP board. The six loops have been closed separately and, although the control loops were independent, the feedback gains used in all the loops are identical. Figure 9 presents some experimental results. The time response shows the signal from one of the force sensors located in the legs, the truss is subjected to an impulse at middle height, without and with control.

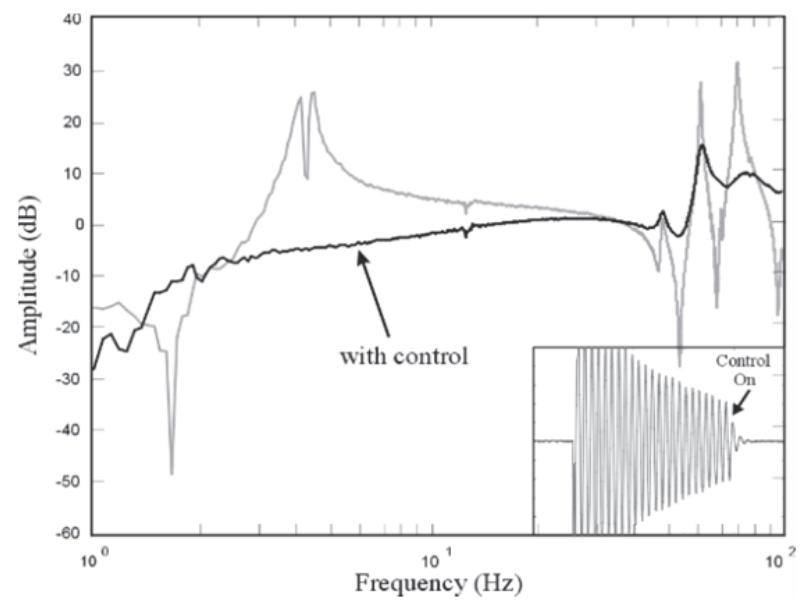

Fig. 9. Experimental time response and frequency response function of the truss mounted on the active interface.

In work [6] the authors propose approach to designing a semi-active stabilization system for space vehicles. In the design of space telescopes, massive primary mirrors are very often used, which can be damaged during the launch phase of the spacecraft. The authors propose a multilevel version of the design of a stabilization system, for stabilizing the structural resonances of the system. Several damping and insulation approaches are discussed, including damping of the viscoelastic restrained layer and damping of the magnetic tunable mass of the mirror segment, passive isolation of spacecraft disturbances and control of the indicators of the active optical telescope. As the actuator of the semi-active stabilization system, the Stuart platform was chosen, and a drive was developed to control the system (Fig. 10).
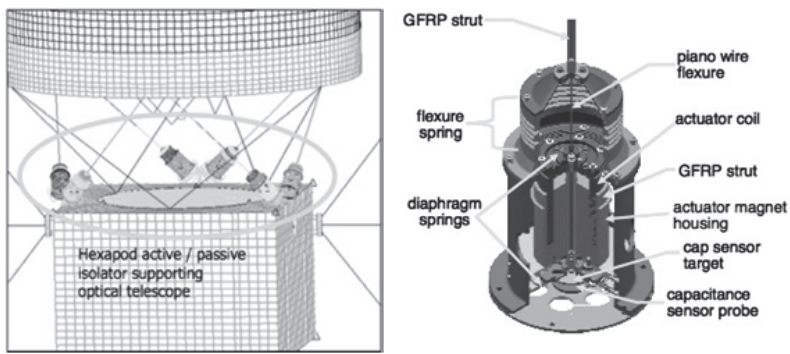

Fig. 10. Implementation of a semi-active stabilization system.

Designed long-stroke actuator for this isolator study has demonstrated stroke capability of $+/-7 \mathrm{~mm}$, which results in significant dynamic range performance and payload steering capability. The actuator design features a voice coil as the active element, diaphragm springs to align the voice coil, and a flexure spring that provides tunable passive isolation capability (see Fig. 11). This design can accommodate a capacitance sensor probe as 
the feedback element, which can be used for telescope positioning in addition to or instead of optical instruments or inertial sensors at the telescope.

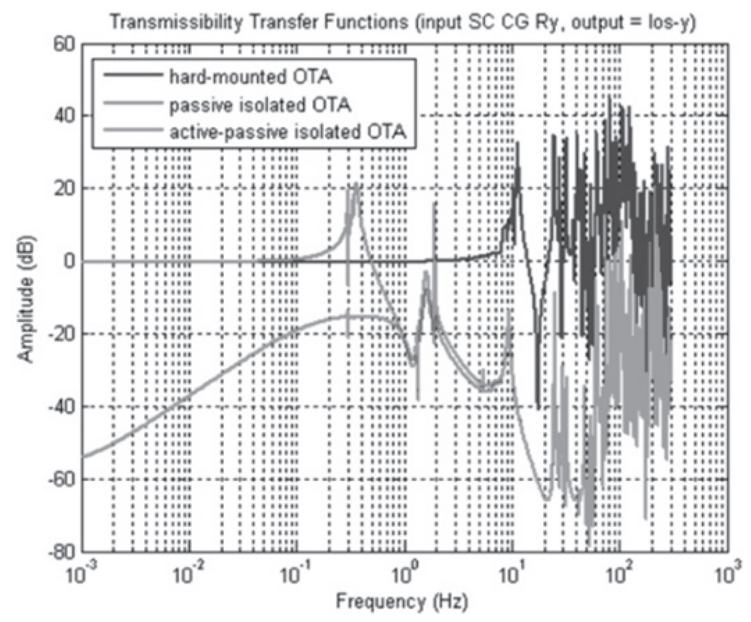

Fig. 11. Transmissibility plots for inputs at spacecraft $C G$ and outputs at telescope LOS for hard- mounted, passive-isolated, and active-passive isolated optical telescope assembly (OTA).

\section{Stabilization system model}

A survey of promising studies on the designing of active stabilization systems has shown that the basic approach to designing is the use of mechanisms with parallel kinematics $[7,8]$. Such mechanisms perceive the load as the spatial trusses, which increases their accuracy characteristics, and the carrying capacity of the entire system. At the given values of the load, the legs of the mechanism are much easier to obtain than the links of a similar mechanism with an open kinematic chain. Thus, a rigid structure with light links makes it possible to significantly increase the speed and accuracy of the system. Therefore, based on the capabilities of such mechanisms, a conclusion was made about the possibility of using such a manipulator as an executive mechanism of the system. In [9], mathematical calculations were carried out, as well as modelling in the MatLab environment, which shows the possibility of fulfilling the requirements for a system of three-dimensional stabilization of optical equipment.

\subsection{Mathematical model of the system}

Let the generalized coordinates of the legs $q_{1}, q_{2}, q_{3}, q_{4}, q_{5}, q_{6} \quad$ be the length $A a, B b, \ldots, F f$ coordinates, respectively. Suppose also that in a fixed Cartesian coordinate system $O X Y Z$ the coordinates of the points $A, B, C, D, E, F$ are equal $\left(X_{1}, Y_{1}, Z_{1}\right)$, and the points $\quad-a, b, c, d, e, f-i\left(X_{1}, Y_{1}, Z_{1}\right), \quad$ where $\quad I \in$ $\{A, B, C, D, E, F\}, i \in\{a, b, c, d, e, f\}$ (Fig. 12).

In the above notation, the solution of the inverse kinematics problem for hexapod (with both four and six degrees of freedom) is given by the following system of equations (1)

$$
q_{i}=\left(X_{A_{i}}-X_{B_{i}}\right)^{2}+\left(Y_{A_{i}}-Y_{B_{i}}\right)^{2}+\left(Z_{A_{i}}-Z_{B_{i}}\right)^{2}
$$

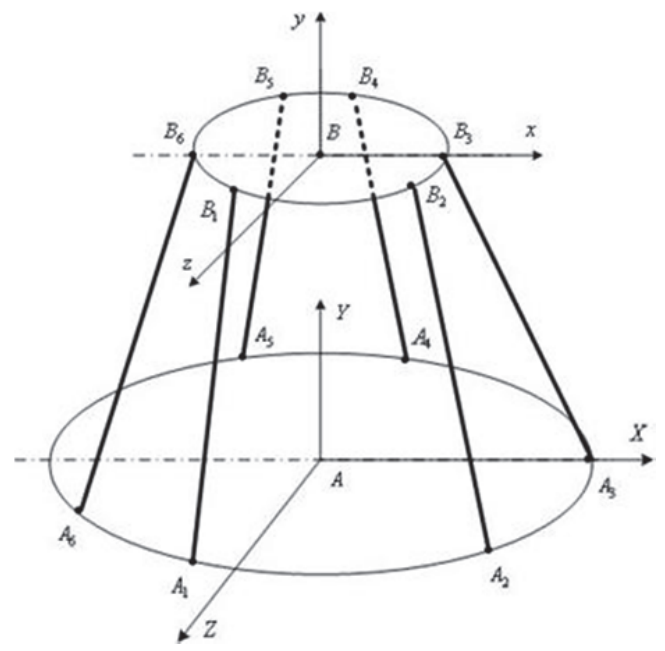

Fig. 12. Schematic representation of the Stewart platform.

The solution of the forward kinematics problem. Let the weightless legs $A_{i} B_{i} \quad i=1,2, \ldots, 6, A B$ consist of two half-stems connected by translational kinematic pairs. The legs $A_{i} B_{i} i=1,2, \ldots, 6$ are attached to the platform at points $B_{i}$ with the help of spherical hinges, and to the base in points $A_{i}$ - using universal joints; The leg $A B$ is connected with the base motionless, and with the platform - with the help of a spherical hinge. Points $B_{i}$ lie on a circle of radius $r$ with centre at point $B$, in which the centre of mass of the platform is located. The base $A_{i}$ is horizontal and the points are located on a circle of radius $R$ with the centre at point $A$. The legs $A_{i} B_{i}$ have lengths $l_{i}\left(l_{i}>0\right)$ and are inclined to the plane of the base at angles $\gamma_{i}\left(0<\gamma_{i}<\frac{\pi}{2}\right)$. The distance between the point $B$ and the base (the plane ) is denoted $h$.

Position of joints $A_{1}, A_{2}, A_{3}, A_{4}, A_{5}, A_{6}$, $B_{1}, B_{2}, B_{3}, B_{4}, B_{5}, B_{6}$ in the coordinates system $A X Y Z$ (Fig. 12) is determined by vectors (2)

$A_{i}=\left(A_{i, 1}, A_{i, 2}, A_{i, 3}\right), B_{i}=\left(B_{i, 1}, B_{i, 2}, B_{i, 3}\right), i \in[1: 6]$.

The position of the platform relative to the base is determined by the Euler angles $\varphi_{1}, \varphi_{2}, \varphi_{3}$, and vector $B=\left(X_{B}, Y_{B}, Z_{B}\right)$. Geometric attitudes between coordinates system $A X Y Z, B x y z$ are given in the transformation matrix $(4 \times 4)-(3)$.

$T=T\left(X_{B}, Y_{B}, Z_{B}, \varphi_{1}, \varphi_{2}, \varphi_{3}\right)=\left[\begin{array}{cccc}a_{1,1} & a_{1,2} & a_{1,3} & X_{B} \\ a_{2,1} & a_{2,2} & a_{2,3} & Y_{B} \\ a_{3,1} & a_{3,2} & a_{3,3} & Z_{B} \\ 0 & 0 & 0 & 1\end{array}\right]$

Where components $a_{i, j}, i, j=1,2,3$ of matrix $T$ are expressed in terms of Euler angles as follows (4)

$$
\begin{gathered}
a_{1,1}=\cos \varphi_{1} \cos \varphi_{3}-\sin \varphi_{1} \cos \varphi_{2} \cos \varphi_{3} \\
a_{1,2}=\sin \varphi_{1} \cos \varphi_{3}+\cos \varphi_{1} \cos \varphi_{2} \sin \varphi_{3} \\
a_{1,3}=\sin \varphi_{2} \sin \varphi_{3} \\
a_{2,1}=-\cos \varphi_{1} \cos \varphi_{3}-\sin \varphi_{1}+\sin \varphi_{1} \cos \varphi_{2} \cos \varphi_{3} \\
a_{2,2}=-\sin \varphi_{1} \sin \varphi_{3}+\cos \varphi_{1} \cos \varphi_{2} \cos \varphi_{3}
\end{gathered}
$$




$$
\begin{gathered}
a_{2,3}=\sin \varphi_{2} \cos \varphi_{3} ; \\
a_{3,1}=\sin \varphi_{1} \sin \varphi_{2} ; \\
a_{3,2}=-\cos \varphi_{1} \sin \varphi_{2} ; \\
a_{3,3}=\cos \varphi_{2} .
\end{gathered}
$$

Thus, position of joint $B_{i}, i \in[1: 6]$ in coordinates system $A X Y Z$ is determined by vector (5)

$$
\left[B_{i}\right]=\left(\begin{array}{c}
{\left[B_{i, 1}\right]} \\
{\left[B_{i, 2}\right]} \\
{\left[B_{i, 3}\right]} \\
1
\end{array}\right)=T\left(\begin{array}{c}
B_{i, 1} \\
B_{i, 2} \\
B_{i, 3} \\
1
\end{array}\right)=T B_{i}
$$

Consequently, generalized coordinates $l_{i}$, as a function of quantities $X_{B}, Y_{B}, Z_{B}, \varphi_{1}, \varphi_{2}, \varphi_{3}$ determined by (6)

$$
\begin{gathered}
l_{i}=l_{i}\left(X_{B}, Y_{B}, Z_{B}, \varphi_{1}, \varphi_{2}, \varphi_{3}\right)= \\
=\sqrt{\sum\left(A_{i, j}-\left[B_{i, j}\right]\right)^{2}}, \\
j=1,2,3, i \in[1: 6] .
\end{gathered}
$$

The notation introduced, the angles between the leg $A_{i} B_{i}, i \in[1: 6]$ and axes of the coordinate system $A X Y Z$ is determined by (7)

$$
\begin{gathered}
\cos \gamma_{i, j}=\cos \gamma_{i, j}\left(X_{B}, Y_{B}, Z_{B}, \varphi_{1}, \varphi_{2}, \varphi_{3}\right)= \\
=\frac{\left[B_{i, j}\right]-A_{i, j}}{l_{i}}, \\
j=1,2,3,
\end{gathered}
$$

where angles $\gamma_{i, 1}, \gamma_{i, 2}, \gamma_{i, 3}$ - angles between leg $A_{i} B_{i}$ and axes $A X, A Y, A Z$.

\subsection{Designing model in Matlab.}

Simulation of the kinematics and dynamics of our system will be performed using Matlab. The Simulink model consists of 3 blocks (Figure 13). In the first block, "Platform", a model of the system is created, which consists of 2 platforms and 6 bars (Fig. 14).

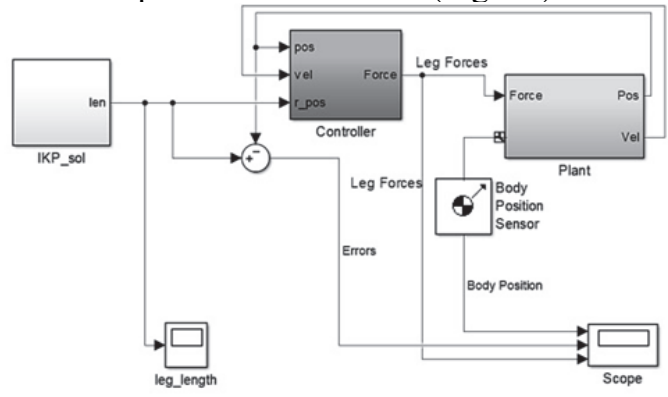

Fig. 13. General model of the system.

The platforms are implemented by the "body" block, in which are given the data on the system mass and coordinates of the points where the hinges connecting the platforms with the links (Fig. 14).

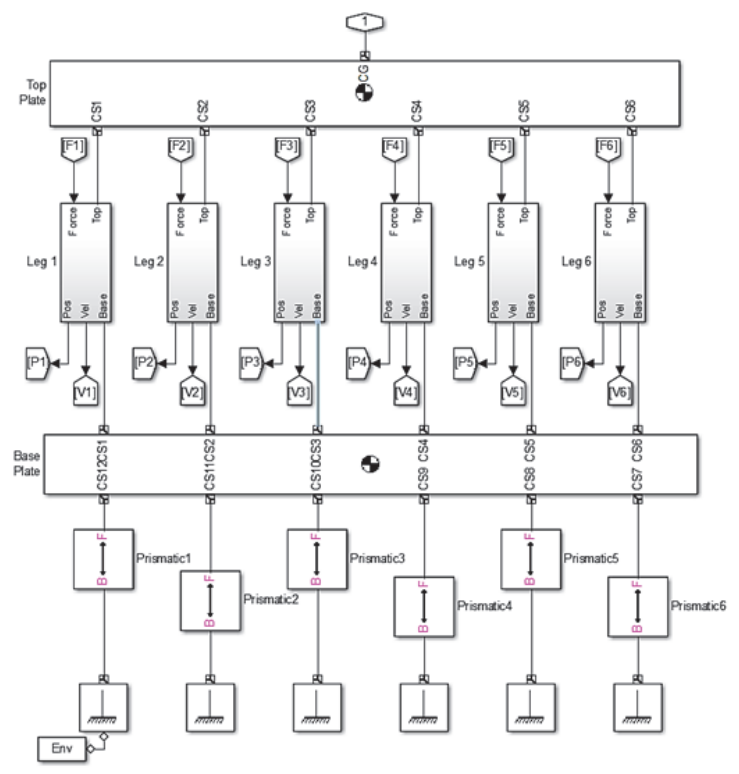

Fig. 14. Hexapod model in Matlab.

As a result of the simulation of the system, we obtain data of the position of the links, the speed, the difference between the desired and actual position of the platform (Fig 15).

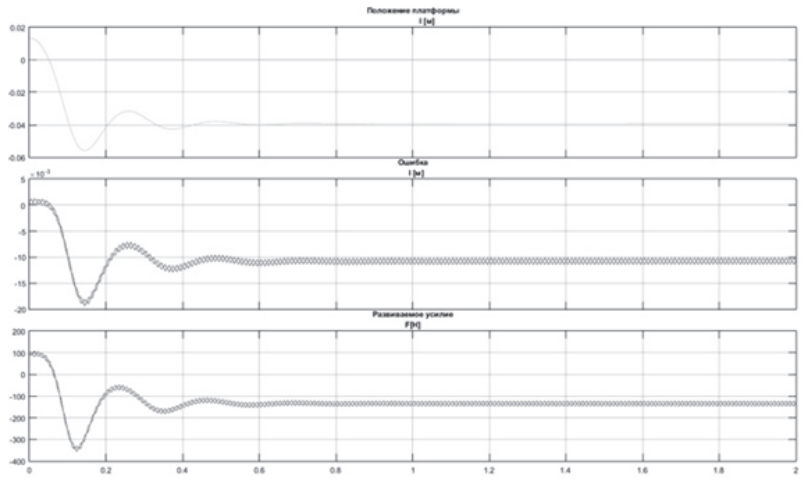

Fig. 15. Modelling results.

The first graph shows the position of each joint of the system. The second graph shows the error generated. The third graph shows the efforts that occur in each boom of the system. The results allowed to put forward demands on the executive modules of the stabilization system and conclude that the hexapod can be used as an executive device for the stabilization system.

\section{Conclusion}

The analysis of the development of high-precision active stabilization and guidance systems shows that it is promising approach to designing such systems is the use of mechanisms with parallel kinematics. It provides the possibility of an even load distribution to the executive modules of the stabilization system. The designed mathematical model of the system made it possible to make demands to the executive modules of the system that would provide the required quality of work. 


\section{References}

1. J.R. Maly et al., Proc. SPIE 8450, Modern Technologies in Spaceand Ground-based Telescopes and Instrumentation II, 845004 (2012)

2. M. Unsal, C. Crane, C. Niezrecki, Florida conference on recent advances in robotics (FCRAR), 25- 26 (2006)

3. M. Elahinia, C. Ciocanel, S. Wang, Smart Materials Research, 2013, Article ID 831017 (2013)

4. C. Wang, X. Xie, Y. Chen, Z. Zhang, 12th International Conference on Recent Advances in Structural Dynamics (2016)

5. Ir. A. Abu Hanieh, A. Preumont, proceedings of the 9th European Space Mechanisms and Tribology Symposium (2001)

6. V. Camelo, A. Bronowicki, S. Simonian, R. Hejal, S. Brennan, 50th AIAA/ASME/ASCE/AHS/ASC Structures, Structural Dynamics, and Materials Conference, 2009, 2637 (2009)

7. Y. Liu, X. Han, J. Han, International Journal of Advanced Robotic Systems, 9 (2) (2012)

8. Y. Song, X. Sun, Appl. Sci. 7, 393 (2017)

9. J.T. Kaganov, A.P. Karpenko, Science and Education of the Bauman MSTU, 11 (2009)

10. J.N. Artemenko, Science and Education of the Bauman MSTU, 01, (2013)

11. J.N. Artemenko, S.N. Sajapin, Science and Education of the Bauman MSTU, 06, (2013)

12. S.V. Volkomorov, J.T. Kaganov, A.P. Karpenko, Informacionnye tehnologii. Prilozhenie, 5, 1-32 (2010)

13. S.V. Volkomorov, A.P. Karpenko, Science and Education of the Bauman MSTU, 12 (2010)

14. S.V. Volkomorov, A.P. Karpenko, A.M. Leletko, Science and Education of the Bauman MSTU, 08 (2010)

15. A.I. Galushkin, S.N. Sajapin, A.V. Sinev, 7 All-Russian Conference "Neirokompjutery i ih primenenie", 184-192 (2001)

16. A.L. Lapikov, V.N. Pashhenko, Science and Education of the Bauman MSTU, 12, (2014) 